Human and Animal Health

Vol.60: e17160368, January-December 2017 http://dx.doi.org/10.1590/1678-4324-2017160368 ISSN 1678-4324 Online Edition

\title{
In Vitro Assessment of Antileishmanial Activity of Natamycin and Nystatin
}

\author{
Arushdeep Sidana ${ }^{1,2}$, Ajeet Kumar Negi $^{3}$, Umar Farooq ${ }^{1,4} *$. \\ ${ }^{I}$ Molecular and Immuno-parasitology Laboratory, Faculty of Applied Sciences and Biotechnology, Shoolini \\ University, Himachal Pradesh, India; ${ }^{2}$ Department of Microbiology, Indira Gandhi Medical College, H.P., India; \\ ${ }^{3}$ Department of Dermatology, Indira Gandhi Medical College, H.P., India; ${ }^{4}$ Faculty of Dentistry, Taif University, \\ Taif.
}

\begin{abstract}
The present study was aimed to evaluate the in vitro antileishmanial activity of four different concentrations of natamycin and nystatin by using MTT 3-(4.5-dimethylthiazol-2-yl)-2.5-diphenyl tetrazolium bromide reduction assay. In vitro antileishmanial activity revealed that the $I C_{50}$ of natamycin $(80.49 \mu \mathrm{g} / \mathrm{ml})$ and nystatin $(105.7 \mu \mathrm{g} / \mathrm{ml})$ was less than that of sodium stibogluconate $(127.9 \mu \mathrm{g} / \mathrm{ml})$, and more than amphotericin $B(18.91 \mu \mathrm{g} / \mathrm{ml})$.
\end{abstract}

Key words: Leishmaniasis, Leishmania donovani, Antileishmanial, Natamycin, Nystatin

\footnotetext{
*Author for correspondence: ufarooq8@gmail.com
} 
Leishmaniasis is a major public health problem in tropical and subtropical countries including India. It is caused by an intracellular obligate protozoan parasite, Leishmania, which spreads through the bite of infected female sand fly. It is estimated that 0.2 to 0.4 million new cases of visceral leishmaniasis (VL) and 0.7 to 1.3 million new cases of cutaneous leishmaniasis (CL) occur every year worldwide ${ }^{1}$. The treatment and control of leishmaniasis involves the administration of pentavalent antimonials, amphotericin B, paromomycin, pentamidine and azoles like ketoconazole ${ }^{2}$. Chemotherapy of leishmaniasis has been challenged by drug resistance, toxicity, variable effectiveness between species and requirement for long durations of treatment ${ }^{3}$. Hence, there is an urgent need to discover or develop new chemotherapeutic agents for the treatment of leishmaniasis which may reduce the unpleasant side-effects associated with conventional drugs and may control the disease safely and efficiently.

Amphotericin B is a polyene antibiotic as well as a broad spectrum antimycotic agent. It is used to cure visceral leishmaniasis cases which are non-responsive to pentavalent antimonials ${ }^{4}$. Despite its $97 \%$ cure rate, it has some drawbacks such as nephrotoxicity that may result in kidney failure ${ }^{5,6}$. Owing to the toxicity of amphotericin B and other available treatments, there is a need to develop agents with antileishmanial activity. Therefore, compounds having structural and functional similarities with amphotericin B may be screened and tested. The antileishmanial action of amphotericin B is believed to be due to its capability to bind ergosterol, a major sterol in Leishmania ${ }^{7}$. Ergosterol is found in the cell membrane of Leishmania and is obligatory to regulate membrane fluidity. It contributes to the organization of membrane domains. Ergosterol is not found in mammals, instead, it is replaced by cholesterol. Therefore, the compounds targeting particularly ergosterol are of great interest to inhibit Leishmania parasite. The antimycotic agent natamycin, a macrolide antifungal agent derived from Streptomyces natalensis and nystatin, a polyene antifungal drug derived from Streptomyces noursei are known to bind with the fungal membrane sterols and are structurally similar to amphotericin B (Figure 1). These two drugs, approved for human use, are broad spectrum antifungal agents and have never been screened against Leishmania donovani. The present study was aimed to evaluate the in vitro antileishmanial potential of natamycin and nystatin against $L$. donovani. 


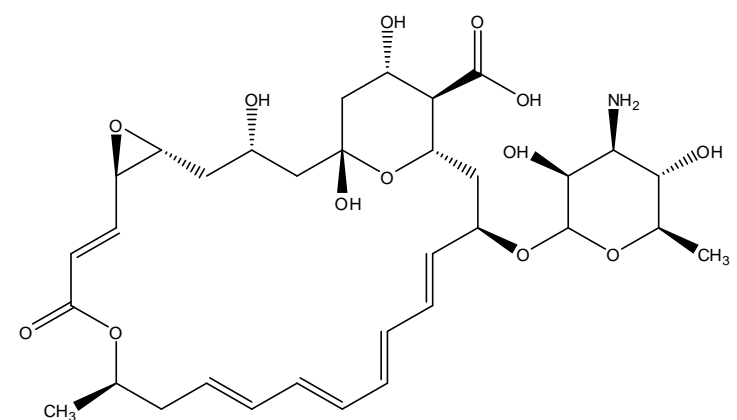

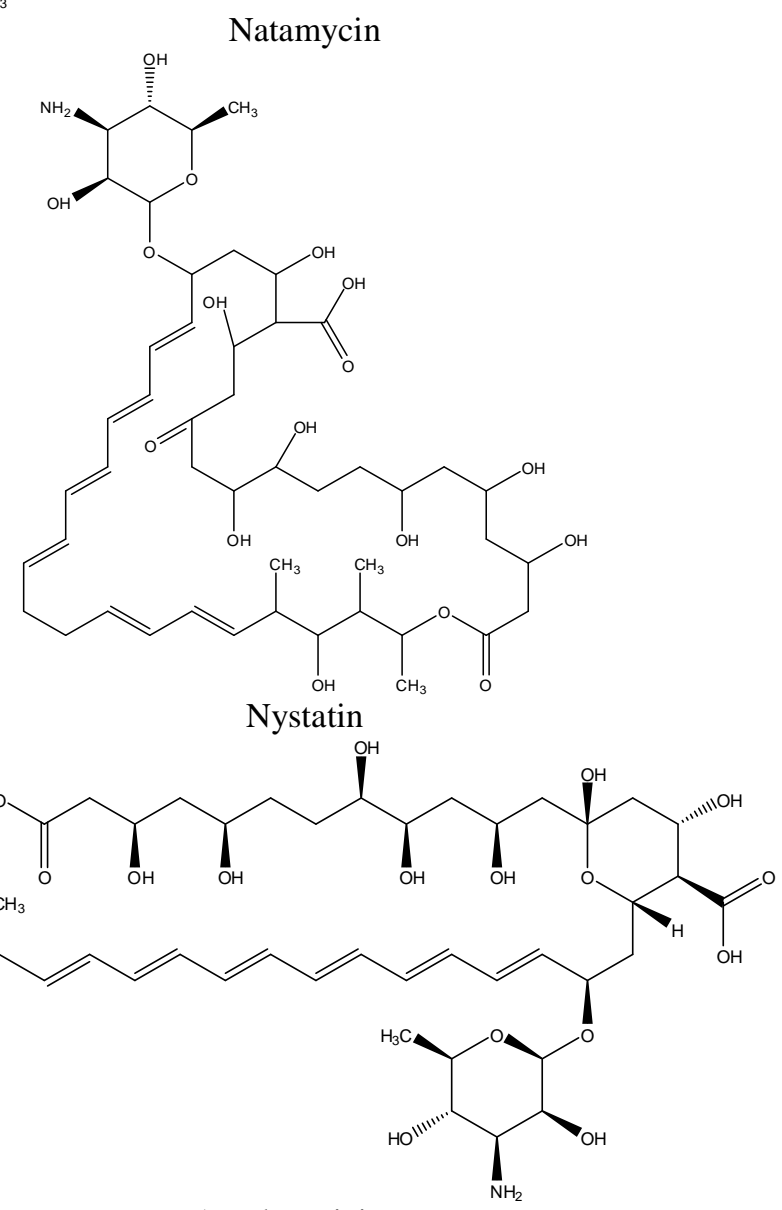

Amphotericin B

Figure 1. Chemical structures of natamycin, nystatin and amphotericin B.

The Axenic culture of $L$. donovani (LdMIPL-1) was maintained at $25^{\circ} \mathrm{C}$ in RPMI1640 (Himedia, Mumbai) medium supplemented with 10\% heat inactivated Fetal Bovine Serum (FBS) (Himedia, Mumbai), streptomycin $(150 \mu \mathrm{g} / \mathrm{ml})$, penicillin G $(100 \mu \mathrm{g} / \mathrm{ml})$ and gentamycin $(150 \mu \mathrm{g} / \mathrm{ml})$ at $\mathrm{pH} 7.2$ For antileishmanial activity, promastigotes of $L$. donovani were sub-cultured in Schneider's Insect Medium (Himedia, Mumbai) supplemented with $10 \%$ FBS, streptomycin $(150 \mu \mathrm{g} / \mathrm{ml})$, penicillin $\mathrm{G}(100 \mu \mathrm{g} / \mathrm{ml})$ and gentamycin $(150 \mu \mathrm{g} / \mathrm{ml})$. The screening was performed in 96-well, flat bottom tissue culture plates (Corning Life Sciences, USA). A cell suspension $(100 \mu \mathrm{l})$ containing $20-30$ million cells $/ \mathrm{ml}$ was poured in each well of the plate. Four different concentrations i.e. 25, 50, 75 and $100 \mu \mathrm{g} / \mathrm{ml}$ of natamycin (Sun Pharma Laboratories, Mumbai) and nystatin (Himedia, Mumbai), dissolved in dimethyl sulfoxide $(\mathrm{DMSO})(<0.025 \% \mathrm{v} / \mathrm{v})$, were added into the culture. The plates were then incubated at $25^{\circ} \mathrm{C}$ for $24-48$ hours. Amphotericin B $(2.5-10.0 \mu \mathrm{g} / \mathrm{ml})$ and 
sodium stibogluconate $(25-100 \mu \mathrm{g} / \mathrm{ml})$ were used as positive controls and cell suspension with $0.025 \%$ DMSO was used as the negative control. The mortality of the promastigotes was assessed by measuring the cleavage of $10 \mathrm{mg} / \mathrm{ml}$ of MTT 3(4.5-dimethylthiazol-2-yl)-2.5-diphenyl tetrazolium bromide ${ }^{8,9}$. The reduction of MTT dye results in the formation of purple formazan. Only the living cells having the enzyme oxidoreductase turn the dye into the purple compound. Thus, the intensity of the purple product formed is directly proportional to the number of living cells in the suspension. In this study, inhibition of promastigotes in the wells with different concentrations of research drugs was assessed by less formazan formed as compared to the negative control in which most of the promastigotes were alive. The absorbance was measured by using ELISA plate reader (BioTek, USA) at $595 \mathrm{~nm}$. The mean percent inhibition was calculated as follows:

$$
\% \text { of inhibition }=\frac{\text { OD control }- \text { OD treated }}{\text { OD control }} \times 100
$$

Each experiment was performed in triplicate with three replicates of each concentration and the results were expressed as mean \pm standard error of the mean (SEM). The $\mathrm{IC}_{50}$ values were calculated using GraphPad Prism 5.02 software. The overall variation in a set of data was analysed by one way analysis of variance (ANOVA). A value of $\mathrm{P}<0.05$ was considered significant.

The results of antileishmanial activity of the two research drugs revealed that both natamycin and nystatin possess significant antileishmanial activity in comparison to sodium stibogluconate while less activity in comparison to amphotericin B (Figure 2-3). The $\mathrm{IC}_{50}$ values of natamycin and nystatin were $80.49 \mu \mathrm{g} / \mathrm{ml}$ and $105.7 \mu \mathrm{g} / \mathrm{ml}$, respectively, which were less than sodium stibogluconate $(127.9 \mu \mathrm{g} / \mathrm{ml})$ and higher than amphotericin B $(18.91 \mu \mathrm{g} / \mathrm{ml})$ (Table 1).

Table 1. Percent inhibition of $L$. donovani promastigotes with four different concentrations of natamycin, nystatin, sodium stibogluconate and amphotericin B.

\begin{tabular}{|c|c|c|c|c|c|}
\hline \multirow{2}{*}{ Drug used $\downarrow$} & \multicolumn{5}{|c|}{ Percent inhibition of L. donovani } \\
\hline & $25 \mu \mathrm{g} / \mathrm{ml}$ & $50 \mu \mathrm{g} / \mathrm{ml}$ & $75 \mu \mathrm{g} / \mathrm{ml}$ & $100 \mu \mathrm{g} / \mathrm{ml}$ & $\mathrm{IC}_{50}(\mu \mathrm{g} / \mathrm{ml})$ \\
\hline Natamycin & $\begin{array}{l}42.27 \\
(1.817)\end{array}$ & $\begin{array}{l}47.53 \\
(1.34)\end{array}$ & $\begin{array}{l}49.59 \\
(0.893)\end{array}$ & $\begin{array}{l}51.07 \\
(1.123)\end{array}$ & 80.49 \\
\hline Nystatin & $\begin{array}{l}38.72 \\
(1.618)\end{array}$ & $\begin{array}{l}42.47 \\
(2.313)\end{array}$ & $\begin{array}{l}46.23 \\
(1.727)\end{array}$ & $\begin{array}{l}50.76 \\
(0.956)\end{array}$ & 105.7 \\
\hline $\begin{array}{l}\text { Sodium } \\
\text { Stibogluconate }\end{array}$ & $\begin{array}{l}30.84 \\
(1.257)\end{array}$ & $\begin{array}{l}36.94 \\
(1.663)\end{array}$ & $\begin{array}{l}42.87 \\
(0.690)\end{array}$ & $\begin{array}{l}47.62 \\
(0.506)\end{array}$ & 127.9 \\
\hline Amphotericin B & $\begin{array}{l}41.28 \\
(1.084) \\
\text { (at } 2.5 \mu \mathrm{g} / \mathrm{ml})\end{array}$ & $\begin{array}{l}41.03 \\
(2.039) \\
(5.0 \mu \mathrm{g} / \mathrm{ml})\end{array}$ & $\begin{array}{l}46.18 \\
(1.935) \\
(7.5 \\
\mu \mathrm{g} / \mathrm{ml})\end{array}$ & $\begin{array}{l}47.52 \\
(1.475) \\
(10.0 \mu \mathrm{g} / \mathrm{ml})\end{array}$ & 18.91 \\
\hline
\end{tabular}

\footnotetext{
*Standard error of the mean (SEM) is shown in brackets.
} 


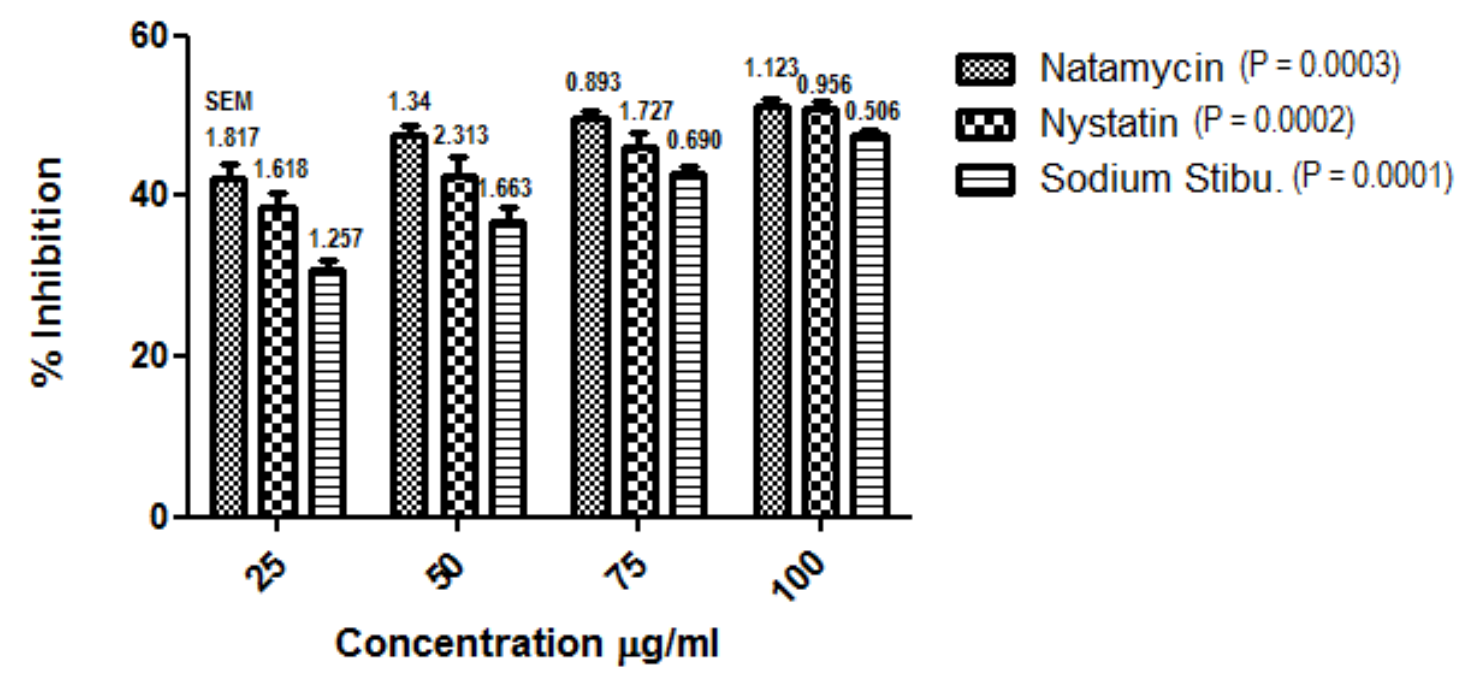

Figure 2. Percent inhibition of $L$. donovani promastigotes by natamycin, nystatin and sodium stibogluconate after 24 hours of incubation at $25^{\circ} \mathrm{C}$ and standard error of the mean. Differences were considered statistically significant $(\mathrm{P}<0.05)$ when comparing the parasites treated with four different concentrations i.e., 25, 50, 75 and $100 \mu \mathrm{g} / \mathrm{ml}$ of natamycin, nystatin and sodium stibogluconate.

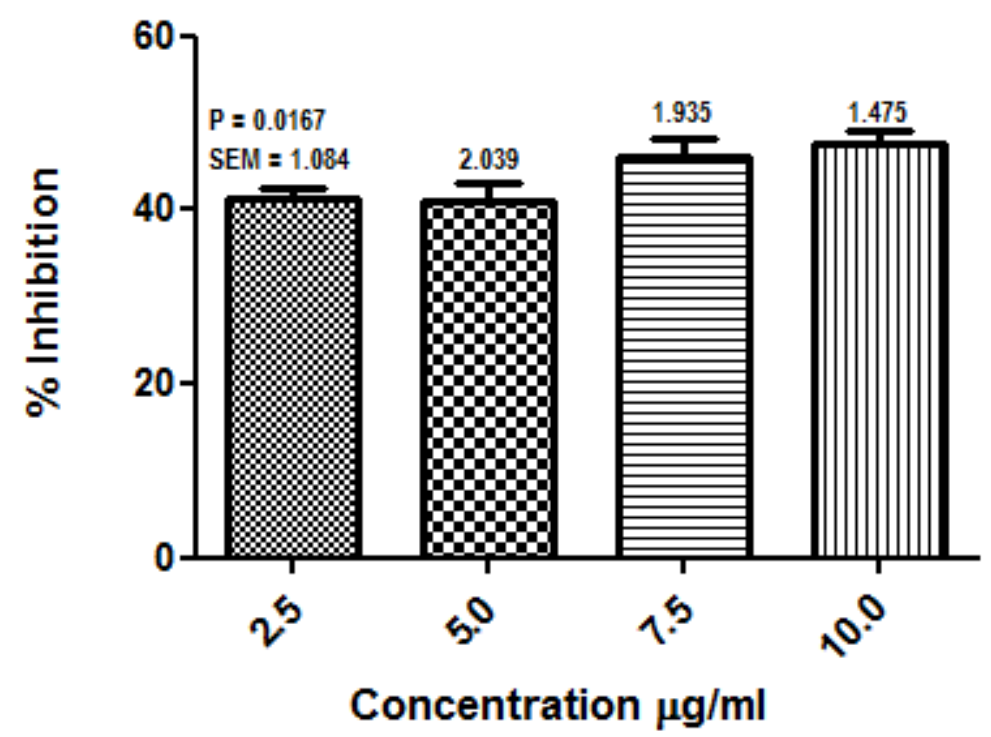

Figure 3. Percent inhibition of L. donovani promastigotes by amphotericin B after 24 hours of incubation at $25^{\circ} \mathrm{C}$. Differences were considered statistically significant $(\mathrm{P}<0.05)$ when comparing the parasites treated with four different concentrations i.e. 2.5, 5.0, 7.5 and $10.0 \mu \mathrm{g} / \mathrm{ml}$ of amphotericin B.

Research is going on worldwide to find new and better antileishmanial agents of natural/synthetic origin. A recent study suggested that the use of antiretroviral drugs individually and in combination with miltefosine was effective against Leishmania infantum. Out of the six tested drugs, efavirenz and delavirdine mesylate individually showed $\mathrm{IC}_{50}$ values of $26.1 \mu \mathrm{M}$ and $136.2 \mu \mathrm{M}$ respectively. On combining efivirenz with miltefosine the $\mathrm{IC}_{50}$ value decreased to $11.8 \mu \mathrm{M}^{10}$. This is a fine idea put in the pipeline which may increase the effectiveness against the parasite while decreasing the common side effects of the previously used drug. In another study, the antileishmanial effect of twelve indolyl-coumarin hybrids was assessed and it was observed that a total of three compounds possessed significant antileishmanial activity with $\mathrm{IC}_{50}$ values in the range of $95-99 \mu \mathrm{g} / \mathrm{ml}{ }^{11}$. Despite the extensive research for better antileishmanial agents, the efficacy of natamycin and nystatin, 
individually or in combination with other drugs, has not been studied against $L$. donovani. Several factors such as mechanism of action against fungi i.e. binding with ergosterol and causing pores in the cell membrane, easy availability, very less toxicity as compared to amphotericin B and approval for human use prompted us to select these two drugs. In the present study, we have evaluated the effectiveness of natamycin and nystatin in vitro against the promastigote forms of $L$. donovani, the most common species of Leishmania found in India. We have observed that nystatin is able to inhibit the growth of parasite within 24 hours of administration with an $\mathrm{IC}_{50}$ value of $105.7 \mu \mathrm{g} / \mathrm{ml}$. In a similar study, the in vitro antileishmanial activities of liposomal nystatin was compared with free nystatin, some amphotericin B formulations and antimycotic azole drugs such as ketoconazole, fluconazole and itraconazole against Leishmania braziliensis, L. infantum and L. tropica. It was observed that liposomal formulations of nystatin were more effective than free nystatin against the promastigotes of $L$. braziliensis but were less effective against $L$. infantum and $L$. tropica ${ }^{12}$. In another study, nystatin has also showed very potent in vitro antileishmanial activity against $L$. major with an $\mathrm{EC}_{50}$ value of $9.76 \mathrm{IU} / \mathrm{ml}{ }^{13} . L$. donovani is the frequent cause of visceral as well as cutaneous leishmaniasis in the endemic areas of India. Most of the patients with visceral leishmaniasis are nonresponsive to glucantime ${ }^{14}$. Therefore, this study was focussed on $L$. donovani for the search of some more effective and safer antileishmanial drug as compared to sodium stibogluconate and amphotericin B. In case of natamycin, we have observed $\mathrm{IC}_{50}$ value of $80.49 \mu \mathrm{g} / \mathrm{ml}$ which is lower than nystatin and sodium stibogluconate. Ophthalmic suspension of natamycin which has been used in the present study has reported to possess potent anti-fungal activity against Fusarium and Aspergillus species ${ }^{15}$. This is the first report of antileishmanial activity of natamycin. The antileishmanial activity shown by standard drug sodium stibogluconate was less as compared to the two research drugs. The calculated $\mathrm{IC}_{50}$ was $127.9 \mu \mathrm{g} / \mathrm{ml}$ which is less than already reported value of $490.00 \mu \mathrm{g} / \mathrm{ml}{ }^{11}$. Amphotericin B has shown very potent antileishmanial activity with an $\mathrm{IC}_{50}$ value of $18.91 \mu \mathrm{g} / \mathrm{ml}$ which is higher as compared to the previously reported values of $0.9,1.9,2.8 \mu \mathrm{g} / \mathrm{ml}$ for promastigotes of L. amazonensis, $L$. chagasi and $L$. amazonensis respectively ${ }^{16,17}$. It may be concluded that the two research drugs natamycin and nystatin used in this study are not as good antileishmanial agents as amphotericin B but their considerable antileishmanial activity and very low toxicity may make these possible competitors of amphotericin B in near future. This may be done by continuous trials of natamycin and nystatin individually, both in combination and in combination with other antileishmanial agents against various species of Leishmania.

An earlier in silico molecular docking study had suggested that natamycin and nystatin were able to inhibit the essential enzymes involved in the purine and pyrimidine metabolism of $L$. donovani ${ }^{18}$. The findings of the present study are in accordance with the in silico study and demonstrates that natamycin and nystatin are more potent antileishmanial agents than sodium stibogluconate, which is the firstline treatment of visceral leishmaniasis all over the world. Further, in vivo study is underway to establish the antileishmanial potential of natamycin and nystatin against L. donovani.

\section{REFERENCES}

1. Rama M, Kumar NV, Balaji S. A comprehensive review of patented antileishmanial agents. Pharm Pat Anal. 2015; 4: 37-56.

2. Kyriazis JD, Aligiannis N, Polychronopoulos P, Skaltsounis AL, Dotsika E. Leishmanicidal activity assessment of olive tree extracts. Phytomedicine. 2013; 20: 275-81. 
3. Camacho Md, Phillipson JD, Croft SL, Solis PN, Marshall SJ, Ghazanfar SA. Screening of plant extracts for antiprotozoal and cytotoxic activities. J Ethnopharmacol. 2003; 89: 18591.

4. Chattopadhyay A, Jafurulla M. A novel mechanism for an old drug: Amphotericin B in the treatment of visceral leishmaniasis. Biochem Biophys Res Commun. 2011; 416: 7-12.

5. Cohen BE. Amphotericin B toxicity and lethality: A tale of two channels. Int $\mathrm{J}$ Pharm. 1998; 162: 95-106.

6.Lemke A, Kiderlen AF, Kayser O. Amphotericin B. Appl Microbiol Biotechnol. 2005; 68: 151-162.

7. Hartsel S, Bolard J. Amphotericin B: New life for an old drug. Trends Pharmacol Sci. 1996; 17: 445-449.

8. Mossman T. Rapid colorimetric assay for cellular growth and survival: Application to proliferation and cytotoxicity assays. J Immunol Methods. 1983; 65: 55-63.

9. Sereno D, Lemesre JL. Use of an enzymatic micromethod to quantify amastigotes stage of Leishmania amazonensis in vitro. Parasitol Res. 1997; 83: 401-403.

10. Costa S, Machado M, Cavadas C, do Céu Sousa M. Antileishmanial activity of antiretroviral drugs combined with miltefosine. Parasitol Res. 2016; Jun 1.

11. Sangshetti JN, Khan FA, Kulkarni AA, Patil RH, Pachpinde AM, Lohar KS, Shinde DB. Antileishmanial activity of novel indolyl-coumarin hybrids: Design, synthesis, biological evaluation, molecular docking study and in silico ADME prediction. Bioorg Med Chem Lett. 2016; 26: 829-835.

12. Piñero J, Martínez E, Ortega A, Del Castillo A, Valladares B. In vitro activity of free and liposomal nystatin against Leishmania species in comparison with several ampbotericin B and azolic formulations. RevistaIbérica de Parasitología. 2003; 63: 93-96.

13. Ali SA, Iqbal J, Nabeel, Khalil Y, Manzoor A, Bukhari I, Ahmad B, Yasinzai MM. Leishmanicidal activity of nystatin (mycostatin): A potent polyene compound. J Pak Med Assoc. 1997; 47: 246-248.

14. Sundar S. Drug resistance in Indian visceral leishmaniasis. Tropical Medicine and Internatinal Health. 2001; 6: 849-854.

15. Lalitha P, Vijaykumar R, Prajna NV, Fothergill AW. In vitro natamycin susceptibility of ocular isolates of Fusarium and Aspergillus species: Comparison of commercially formulated natamycin eye drops to pharmaceutical-grade powder. J Clin Microbiol. 2008; 46: 3477-3478.

16. Braga FG, Bouzada MLM, Fabri RL, Matos MO, Moreira FO, Scio E. Antileishmanial and antifungal activity of plants used in traditional medicine in Brazil. J Ethnopharmacol. 2007; 111: 396-402.

17. Pereira IO, Marques MJ, Pavan AL, Codonho BS, Barbieri CL, Beijo LA, Doriguetto AC, D'Martin EC, dos Santos MH. Leishmanicidal activity of benzophenones and extracts from Garcinia brasiliensis Mart. Fruits. Phytomedicine. 2010; 17: 339-345.

18. Sidana A, Farooq U. Natamycin and nystatin: Two novel inhibitors of Leishmania donovani essential enzymes. Int J Biol Pharm Alld Sc. 2015; 4(7): 4534-4544. 